\title{
Inhibition of placental mTOR signaling provides a link between placental malaria and reduced birthweight
}

\author{
Kris Genelyn Dimasuay ${ }^{1,2^{*}}$, Elizabeth H. Aitken ${ }^{1}$, Fredrick Rosario ${ }^{3}$, Madi Njie ${ }^{1}$, Jocelyn Glazier ${ }^{4}$, \\ Stephen J. Rogerson ${ }^{1,5}$, Freya J. I. Fowkes ${ }^{2,6,7}$, James G. Beeson ${ }^{1,2,8}$, Theresa Powell ${ }^{3,9}$, Thomas Jansson ${ }^{3+}$ \\ and Philippe Boeuf ${ }^{1,2,5^{* \dagger}}$ (D)
}

\begin{abstract}
Background: Placental Plasmodium falciparum malaria can trigger intervillositis, a local inflammatory response more strongly associated with low birthweight than placental malaria infection alone. Fetal growth (and therefore birthweight) is dependent on placental amino acid transport, which is impaired in placental malaria-associated intervillositis. Here, we tested the hypothesis that mechanistic target of rapamycin (mTOR) signaling, a pathway known to regulate amino acid transport, is inhibited in placental malaria-associated intervillositis, contributing to lower birthweight.
\end{abstract}

Methods: We determined the link between intervillositis, mTOR signaling activity, and amino acid uptake in tissue biopsies from both uninfected placentas and malaria-infected placentas with and without intervillositis, and in an in vitro model using primary human trophoblast (PHT) cells.

Results: We demonstrated that (1) placental mTOR activity is lower in cases of placental malaria with intervillositis, (2) placental mTOR activity is negatively correlated with the degree of inflammation, and (3) inhibition of placental mTOR activity is associated with reduced placental amino acid uptake and lower birthweight. In PHT cells, we showed that (1) inhibition of mTOR signaling is a mechanistic link between placental malaria-associated intervillositis and decreased amino acid uptake and (2) constitutive mTOR activation partially restores amino acid uptake.

Conclusions: Our data support the concept that inhibition of placental mTOR signaling constitutes a mechanistic link between placental malaria-associated intervillositis and decreased amino acid uptake, which may contribute to lower birthweight. Restoring placental mTOR signaling in placental malaria may increase birthweight and improve neonatal survival, representing a new potential therapeutic approach.

Keywords: Intervillositis, Fetal growth restriction, System A transporter, Deptor

\section{Background}

One in six infants worldwide are born with low birthweight $(<2500 \mathrm{~g})$, which is the main risk factor underlying $80 \%$ of neonatal deaths [1]. The World Health Organization reaffirmed reducing the prevalence of low birthweight by $30 \%$ by 2025 as a global health priority. Malaria in pregnancy is a leading cause of low birthweight and is responsible

\footnotetext{
*Correspondence: genelyn_dimasuay@yahoo.com;

philippe.boeuf@burnet.edu.au

${ }^{\dagger}$ Equal contributors

'Department of Medicine at Royal Melbourne Hospital, The University of Melbourne, Parkville 3004, VIC, Australia

Full list of author information is available at the end of the article
}

for $\sim 900,000$ low birthweight deliveries and $\sim 200,000$ infant deaths annually [2]. Despite control measures, Plasmodium falciparum malaria still affects about 85 million pregnancies each year [3]. Little is known about the mechanistic link between malaria in pregnancy and low birthweight.

Malaria in pregnancy can lead to placental malaria characterized by the sequestration of $P$. falciparum-infected erythrocytes in the maternal intervillous blood space of the placenta. This can trigger the recruitment and activation of maternal immune cells, resulting in a local inflammatory response termed intervillositis. Placental 
malaria-associated intervillositis is more strongly associated with low birthweight than placental malaria without intervillositis [4]. The underlying mechanisms linking placental malaria-associated intervillositis and decreased birthweight are unknown, which hinders the development of intervention strategies aimed at improving the birthweight of infants born to malaria-infected women. Current malaria control strategies such as insecticide-treated bed nets, intermittent preventative malaria treatment of pregnant women, and supplementation of their diet have limited efficacy at improving birthweight $[5,6]$. There is a significant and urgent need for additional interventions aimed directly at improving birthweight that can complement existing malaria control strategies.

Placental nutrient transfer controls fetal nutrient availability, which is a key determinant of fetal growth (and therefore birthweight). The placental capacity to transfer nutrients is highly dependent on the expression and function of nutrient transporters in the syncytiotrophoblast (the nutrient transporting epithelium of the human placenta) [7]. System A is a group of amino acid transporters that mediate the uptake of non-essential neutral amino acids. Decreased placental System A activity has been associated with decreased birthweight both in humans $[8,9]$ and in animal models $[10,11]$. Importantly, the magnitude of the decrease in placental System A activity correlates with the severity of fetal growth restriction in women [8] and placental System A activity is decreased before fetal growth restriction is observed in animal models $[10,11]$. This suggests that downregulation of placental System A activity directly contributes to reduced birthweight. We previously demonstrated that placental malaria-associated intervillositis reduced both the expression and activity of System A transporters, and that System A activity and birthweight are positively correlated in placental malaria [12]. However, the mechanism(s) by which placental malaria-associated intervillositis impacts placental System A activity is unknown.

The mechanistic target of rapamycin (mTOR) signaling pathway is a nutrient-sensing pathway that regulates cell growth, proliferation, and metabolism in response to hormones, growth factors, and nutrient availability (Additional file 1: Table S1). It exists as two protein complexes: mTOR complex 1 (mTORC1), the master regulator of protein translation and cell growth and proliferation; and mTOR complex 2 (mTORC2), which regulates cytoskeletal organization and cellular metabolism. mTOR is expressed in the placental syncytiotrophoblast where it regulates amino acid uptake by influencing the trafficking of transporters to the plasma membrane [13]. Upstream signals such as amino acids, growth factors, free fatty acids, oxygen, and cytokines have been shown to influence placental mTOR signaling activity $[14,15]$. In animal models, placental mTOR inhibition was associated with decreased placental amino acid transport [11]. Similar findings were observed in human fetal growth restriction of causes unrelated to placental malaria [16]. These data suggest that placental mTOR signaling influences fetal growth and birthweight by regulating transplacental nutrient transport in response to maternal signals.

Here, we provide for the first time evidence that inhibition of mTOR signaling is a mechanistic link between placental malaria-associated intervillositis and decreased amino acid transport, contributing to lower birthweight. Our findings open novel avenues of research to develop interventions targeting placental mTOR signaling to improve birthweight and neonatal health in malaria-exposed populations.

\section{Methods}

Detailed materials and methods can be found in Additional file 1.

\section{Collection of placental samples}

The College of Medicine Research Ethics Committee, University of Malawi, approved this study. Written informed consent was obtained from primiparous Malawian women. Placental villous tissue biopsies collected after delivery were grouped based on histology as described previously [4]: uninfected (no malaria, no intervillositis), placental malaria without intervillositis, and placental malaria with intervillositis. Table 1 summarizes the clinical characteristics of the study subjects.

\section{Primary trophoblast cell culture}

Placental villous tissue samples were collected from healthy women with normal term pregnancies following written informed consent as approved by the Colorado Multiple Institutional Review Board (COMIRB-14-1073). Primary human trophoblast (PHT) cells were isolated by trypsin digestion and Percoll centrifugation as originally described [17] with modifications [18]. Syncytialization was assessed by human chorionic gonadotropin secretion. Cell viability was assessed by lactate dehydrogenase release.

\section{Monocyte-conditioned media}

Conditioned media were prepared as previously described [12] with modifications. CD14 ${ }^{+}$cells were cultured in 1:1 mixture of Dulbecco's modified Eagle's medium (DMEM, Sigma-Aldrich) and Ham's F-12 nutrient mixture (Invitrogen) supplemented with $10 \%$ fetal bovine serum and with penicillin/streptomycin.

\section{Small interfering RNA transfection}

PHT cells were transfected with $10 \mathrm{nM}$ DEPTOR small interfering RNA (siRNA; SASI_Hs01_00204344, Sigma- 
Table 1 Clinical characteristics of study subjects

\begin{tabular}{|c|c|c|c|c|}
\hline & Uninfected & Placental malaria without intervillositis & Placental malaria with intervillositis & $P$ value \\
\hline Subjects & 17 & 7 & 14 & \\
\hline \multirow[t]{2}{*}{ Age (years) } & 19 & 18 & 20 & 0.61 \\
\hline & $(18-19)$ & $(17-21)$ & $(18-21)$ & \\
\hline \multirow[t]{2}{*}{ Gestational age (weeks) } & 40 & 40 & 40 & 0.87 \\
\hline & $(38-40)$ & $(38-40)$ & $(38-40)$ & \\
\hline \multirow[t]{2}{*}{ Maternal weight at enrollment (kg) } & 56 & 56 & 56 & 0.77 \\
\hline & $(49.5-58.5)$ & $(52-60)$ & $(52-56)$ & \\
\hline \multirow[t]{2}{*}{ Percentage of monocytes } & 0 & 2.2 & 8.6 & 0.0001 \\
\hline & & $(1.2-3)$ & $(6.6-10.8)$ & \\
\hline \multirow[t]{2}{*}{ Parasitemia (\%) } & 0 & 0.41 & 1.2 & 0.0001 \\
\hline & & $(0.21-0.83)$ & $(0.66-11.2)$ & \\
\hline \multirow[t]{2}{*}{ Birthweight (kg) } & 3.0 & 2.8 & 2.9 & 0.54 \\
\hline & $(2.7-3.5)$ & $(2.6-3.1)$ & $(2.6-3.0)$ & \\
\hline \multirow[t]{2}{*}{ Placental weight (g) } & 500 & 530 & 495 & 0.80 \\
\hline & $(430-550)$ & $(460-560)$ & $(420-580)$ & \\
\hline \multirow[t]{2}{*}{ Fetal-to-placental weight ratio } & 5.95 & 5.71 & 5.89 & 0.48 \\
\hline & $(5.2-6.48)$ & $(4.11-6.09)$ & $(5.26-6.20)$ & \\
\hline
\end{tabular}

Values are presented as median and interquartile range

Aldrich) or with Scramble siRNA control using Lipofectamine RNAiMax transfection reagent (Thermo Scientific) according to the manufacturer's protocol.

\section{Amino acid uptake}

System A activity was assessed by measuring the $\mathrm{Na}^{+}$-dependent uptake of ${ }^{14} \mathrm{C}$-methyl-aminoisobutyric acid (MeAIB) as previously described [13].

\section{Western blot}

Placental homogenates and PHT cell lysates were loaded and proteins separated on 4-12\% Bis-Tris gels (Invitrogen) or $12 \%$ Mini-Protean protein gels (Bio-Rad) and transferred onto $0.20-\mu \mathrm{m}$ nitrocellulose membrane (GE Healthcare) or polyvinylidene fluoride membrane (BioRad). Membranes were incubated with primary antibodies: rabbit anti-4EBP-1, anti-phospho-4EBP-1 $\left(\mathrm{Thr}^{37 / 46}\right)$, antiribosomal protein $\mathrm{S} 6$, anti-phospho-ribosomal protein $\mathrm{S} 6$ $\left(\mathrm{Ser}^{235 / 236}\right)$, anti-Akt, anti-phospho-Akt $\left(\mathrm{Ser}^{473}\right)$, and mouse anti- $\beta$-actin (Sigma-Aldrich). Horseradish peroxidaseconjugated secondary anti-rabbit and anti-mouse antibodies (Cell Signaling) were used as secondary antibodies. Proteins were visualized using chemiluminescence detection. Densitometry was performed using NIH's ImageJ software.

\section{Cytokine analysis}

Cytokine profiles in monocyte-conditioned media were analyzed by a multiplexed bead-based immunoassay using a panel of antibodies against human inflammatory cytokines (BD Biosciences) according to manufacturer's instructions.

Data presentation and statistical analysis

Data are presented as medians and scatter plots or medians and inter-quartile range. Data were analyzed and graphs designed using Prism 5 software (Graph Pad). Two-group comparisons were made using Mann-Whitney test and three-group comparisons using Kruskal-Wallis test. Spearman's correlation test was used to assess correlations with 95\% confidence interval.

\section{Results}

Placental mTOR signaling is inhibited in placental malaria-associated intervillositis

We aimed to assess the impact of $P$. falciparum infection and intervillositis on mTOR signaling in human placenta using well-established mTOR functional readouts as summarized in Additional file 1: Table S1. Placental mTOR signaling activity was measured by quantifying the ratio of phosphorylated-to-total protein expression levels of downstream targets of both mTORC1 ( $\mathrm{rpS6}^{\mathrm{Ser} 235 / 236}$ and 4E-BP1 $1^{\text {Thr37/46 }}$ ) and mTORC2 (Akt ${ }^{\text {Ser473}}$ ) (Fig. 1a). The activity of both mTORC1 and mTORC2 (Fig. 1b) was lower in women with placental malaria-associated intervillositis compared to uninfected placentas (rpS6: $P=0.01$; 4EBP-1: $P=0.03$; Akt: $P=0.0006)$ and to infected placentas without intervillositis for Akt signaling (Akt: $P=0.02$ ). These results are consistent with a causal 

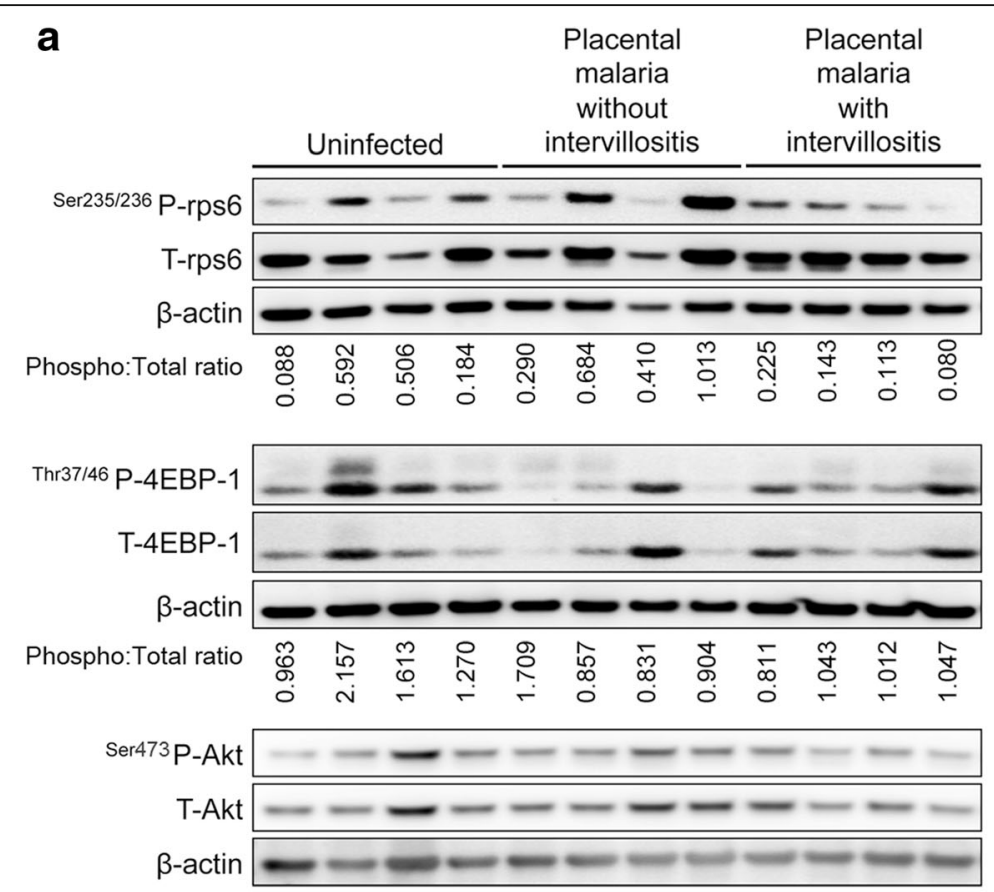

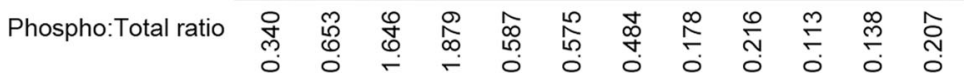

b

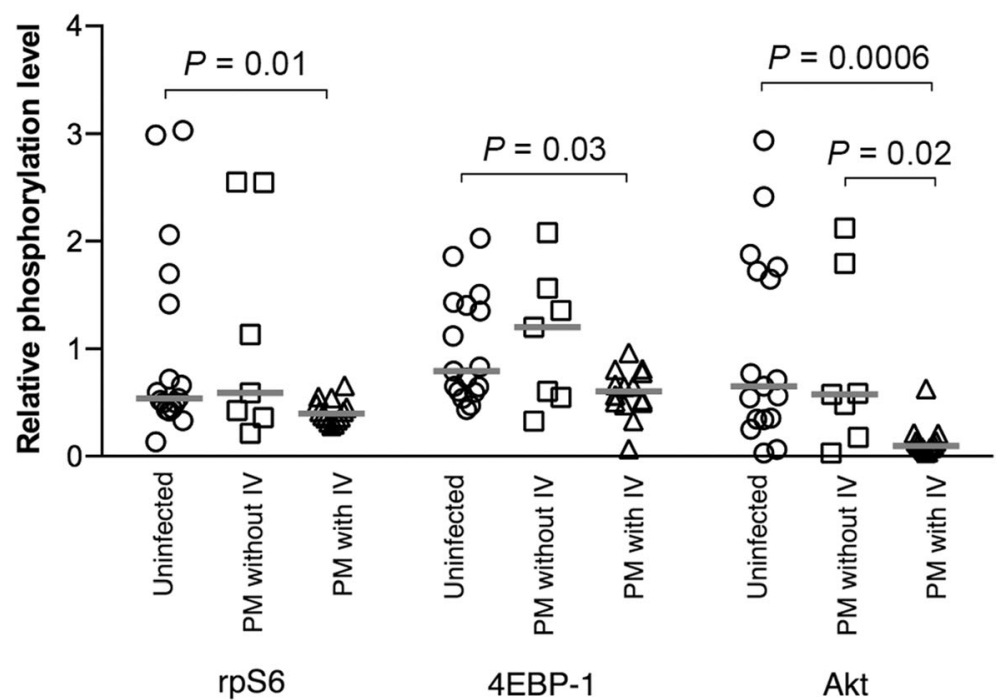

Fig. 1 Placental malaria with intervillositis is associated with inhibition of placental mTOR signaling. mTOR activity was determined as the phosphorylated-to-total protein expression levels of rps6, 4EBP-1, and Akt in placentas grouped into uninfected $(n=17)$, placental malaria without intervillositis $(n=7)$, and placental malaria with intervillositis $(n=14)$. a Representative Western blot. b mTOR signaling activity is inhibited in placental malaria with intervillositis. PM placental malaria, IV intervillositis

link between placental malaria-associated intervillositis and inhibition of mTOR signaling. This was further supported by the negative correlation between mTOR signaling activity and the severity of intervillositis measured as the percentage of monocytes in the intervillous space (Table 2 and Additional file 2: Figure S1).
Inhibition of placental mTOR signaling is associated with impaired amino acid transport and reduced birthweight Inhibition of mTOR signaling may lead to reduced birthweight by impacting amino acid transport. We determined the relationship between placental mTOR signaling activity, amino acid transport, and birthweight. mTOR signaling 
Table 2 Correlation between mTOR signaling activity and percentage of monocyte, amino acid uptake and birthweight

\begin{tabular}{llll}
\hline & \multicolumn{3}{l}{ mTOR signaling activity $^{\mathrm{a}}$} \\
\cline { 2 - 4 } & rps6 & 4EBP-1 & Akt \\
\hline Monocytes (\%) & $r=-0.34$ & $r=-0.34$ & $r=-0.59$ \\
& $(-0.60$ to -0.02$)$ & $(-0.60$ to -0.02$)$ & $(-0.77$ to -0.32$)$ \\
& $P=0.01$ & $P=0.01$ & $P=0.0001$ \\
Amino acid uptake & $r=0.28$ & $r=0.40$ & $r=0.44$ \\
(System A activity) & $(-0.07$ to 0.57$)$ & $(0.07$ to 0.65$)$ & $(0.12$ to 0.68$)$ \\
& $P=0.04$ & $P=0.008$ & $P=0.004$ \\
Birthweight & $r=-0.03$ & $r=0.27$ & $r=0.25$ \\
& $(-0.36$ to 0.30$)$ & $(-0.07$ to 0.55$)$ & $(-0.08$ to 0.54$)$ \\
& $P=0.42$ & $P=0.05$ & $P=0.05$ \\
\hline
\end{tabular}

Correlation analyses are presented as $r$ score and $95 \%$ confidence interval. These analyses include all participants $(n=38)$.

aPhosphorylated-to-total protein expression ratio

showed a positive correlation with our previous microvillous plasma membrane amino acid uptake data [12] measured as System A activity (Table 2 and Additional file 2: Figure S1). This is consistent with our previous reports demonstrating that mTOR signaling is a major regulator of placental System A activity [19, 20], an important contributor to fetal growth. Importantly, we found a positive correlation between mTOR signaling activity and birthweight (Table 2 and Additional file 2: Figure S1). Birthweight was also marginally positively correlated with the degree of intervillositis ( $n=38 ; \mathrm{r}=-0.27 ; P=0.093$ ). Collectively, these data are consistent with the model that placental malaria-associated intervillositis decreases placental mTOR signaling, which reduces amino acid uptake and birthweight.

\section{Intervillositis inhibits mTOR signaling and System A amino acid transport activity in cultured primary human trophoblast cells}

To establish a causal link between inhibition of mTOR signaling and reduced System A activity in placental malaria-associated intervillositis, we adapted our published protocol for studies of placental responses to placental malaria-associated intervillositis in BeWo cells for use in PHT cells [12]. Placental malaria-associated intervillositis was modeled using the conditioned medium from a monocyte/P. falciparum-infected erythrocyte coculture (herein "infected conditioned medium"). This conditioned medium displayed high levels of inflammatory cytokines such as IL-1 $\beta$, IL-6, IL-8, TNF, and IL-10 (Additional file 3: Figure S2). Conditioned medium from a monocyte/uninfected erythrocyte co-culture (herein "uninfected conditioned medium") and culture medium (herein "control") were used as controls.

Both mTORC1 and mTORC2 signaling activity were significantly lower in PHT cells exposed to infected conditioned medium compared to PHT cells exposed to uninfected conditioned medium (4EBP-1: $P=0.04$; Akt: $P=0.09$ ) or to control medium (rps6: $P=0.01$; 4EBP-1: $P=0.008$; Akt: $P=0.002$ ) (Fig. 2). This inhibition of mTOR signaling activity was paralleled by reduced System A activity in PHT cells exposed to infected conditioned medium compared to PHT cells exposed to uninfected conditioned medium $(P=0.04)$ or to control medium $(P=0.03)$ (Fig. 2c). The viability of PHT cells was unaffected by exposure to infected conditioned media (Additional file 4: Figure S3). Collectively, these data are in agreement with our observations in women with placental malaria (Fig. 1), supporting the hypothesis placental malaria-associated intervillositis inhibits mTOR signaling, which results in reduced System A activity.

\section{Activation of mTOR signaling partially restores System A activity in response to placental malaria-associated intervillositis}

To firmly establish placental mTOR signaling inhibition as a mechanistic link between placenta malaria-associated intervillositis and reduced System A activity, we determined the effect of constitutive mTOR signaling activation on System A activity in PHT cells exposed to infected conditioned medium. To constitutively activate mTOR signaling, we silenced DEPTOR, the endogenous inhibitor of mTOR [21], using siRNA. DEPTOR silencing reduced DEPTOR protein expression by approximately 40\% ( $P=0.004)$ (Fig. 3a) without affecting cell differentiation and viability (Additional file 4: Figure S3). As expected, DEPTOR silencing significantly increased mTORC1 signaling activity (rps6: $+44 \%, P=0.008$; 4 EBP1: $+60 \%, P=0.004$; Figs $3 \mathrm{~b}, \mathrm{c})$ and upregulated System A activity $(+66 \%, P=0.009$; Fig. $3 d)$ [13]. This is consistent with previous reports that silencing of DEPTOR activates mTORC1 [21].

We quantified the decrease in System A activity induced by the infected conditioned medium in PHT cells transfected with DEPTOR siRNA versus PHT cells transfected with Scramble siRNA. In cells transfected with Scramble siRNA, System A activity decreased by $72 \%$ in response to the infected conditioned medium whereas System A activity in DEPTOR-silenced cells was decreased by only $50 \%$ (Fig. 3e). This demonstrated that mTOR signaling activation by DEPTOR silencing attenuated the inhibition of System A activity in response to infected conditioned medium by approximately 30\% $(P=0.004)$. This suggests that placental mTOR activation could partially restore placental amino acid uptake in response to placenta malaria-associated intervillositis.

\section{Discussion}

Reducing the incidence of low birthweight remains a global health priority to minimize neonatal morbidity and mortality, impaired infant growth and cognitive development, and 


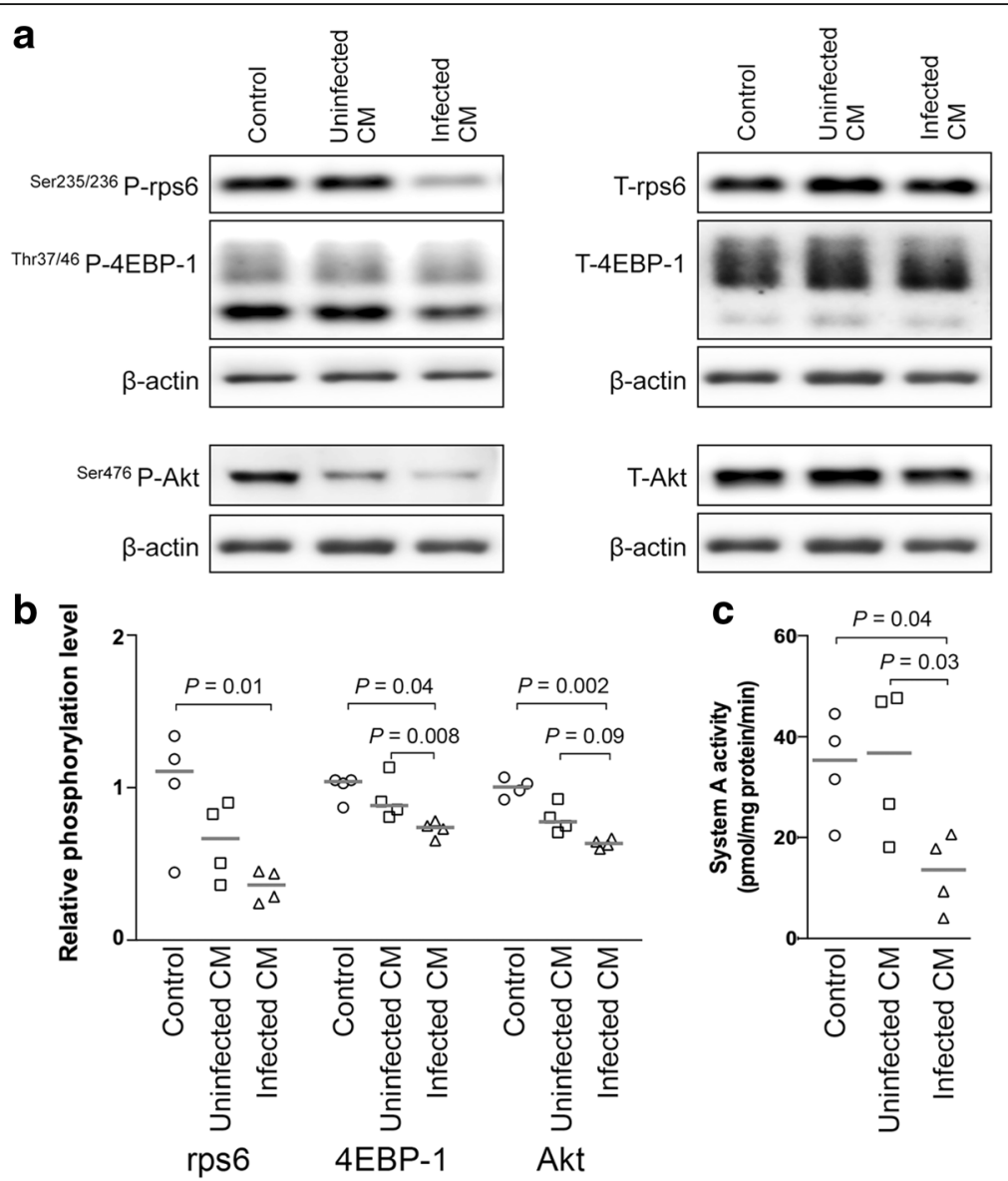

Fig. 2 Placental mTOR signaling inhibition in an in vitro model of placental malaria-associated intervillositis. mTOR activity was determined as the phosphorylated-to-total protein expression levels of rps6, 4EBP-1, and Akt in primary human trophoblast cells exposed to infected conditioned medium, uninfected conditioned medium, and culture medium alone. a Representative Western blot. b mTOR signaling activity and $\mathbf{c}$ System A activity are decreased in primary human trophoblast cells exposed to infected conditioned medium. $n=4$ placentas. CM conditioned medium, Control culture medium alone

chronic diseases later in life [22]. Understanding the pathogenesis of reduced birthweight in placental malaria will provide the foundation for the development of novel interventions that complement existing malaria control approaches to directly improve fetal growth and prevent poor neonatal and pregnancy outcomes. In this study, we provide the first evidence for a role of placental mTOR signaling in decreased placental amino acid uptake and lower birthweight in placental malariaassociated intervillositis. Specifically, we demonstrate that mTOR signaling is inhibited in placental malariaassociated intervillositis and in cultured PHT cells exposed to malaria-infected conditioned media. Furthermore, we provide evidence that mTOR signaling inhibition mechanistically links intervillositis to decreased amino acid transport.

The mTOR signaling pathway has been proposed to play a central role in placental nutrient sensing [23]. This model proposes that the placenta regulates its nutrient transport function to match maternal supply and fetal demand by responding to upstream maternal signals and modulating placental function, including transplacental amino acid transport [23, 24]. In placental malariaassociated intervillositis, maternal mononuclear cells activated by infected erythrocytes in the intervillous space release inflammatory mediators that create a distinct milieu characterized by elevated levels of cytokines and chemokines such as IFN- $\gamma$, TNF, IL-10, MCP-1, MIP-1 $\alpha$, IL-8, CCL2, and CCL3 [25-27]. Some of these circulating inflammatory mediators could be responsible for the inhibition of placental mTOR signaling we observed in the placentas of women with placental malaria with intervillositis. The key role of intervillositis in causing inhibition of placental mTOR signaling is supported in our current study by the strong negative correlation between mTOR signaling activity and the degree of intervillositis.

Our ex vivo results reported here and in our previous publication [12] have demonstrated that placental malaria- 

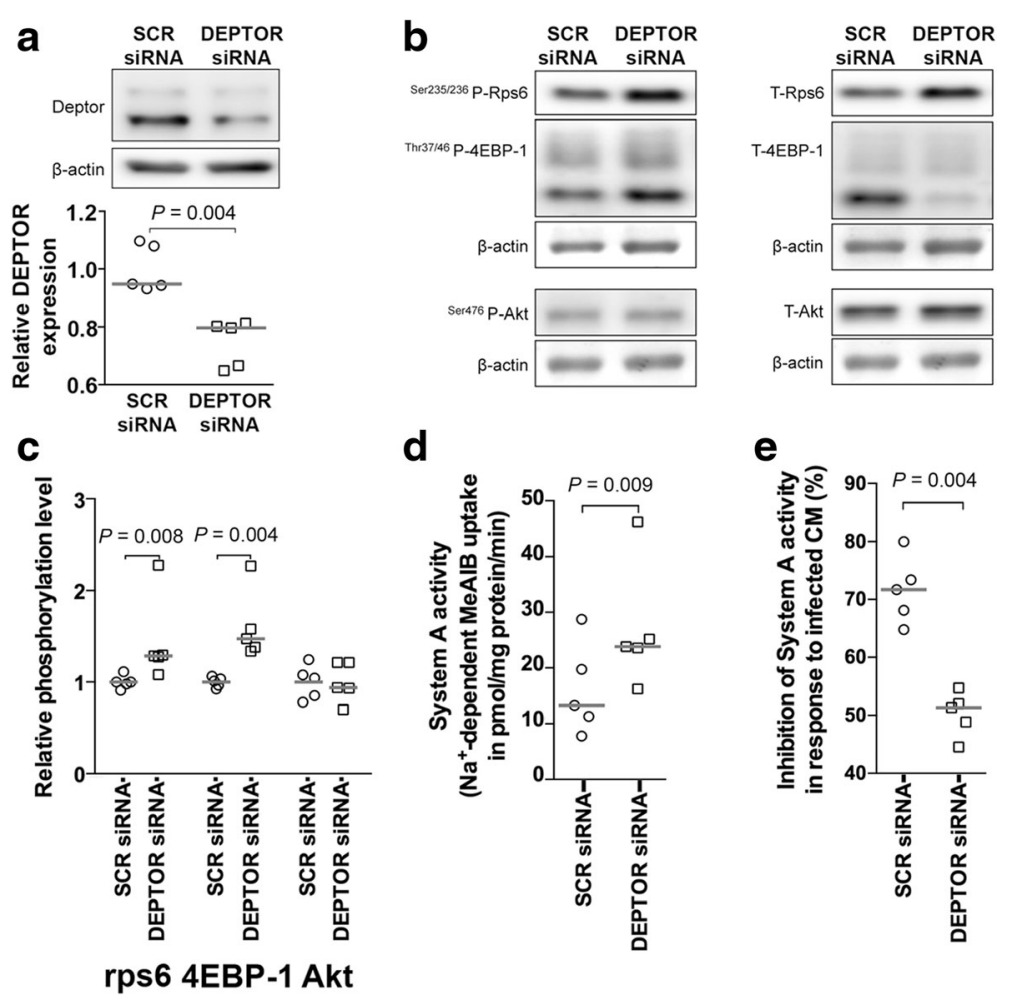

d

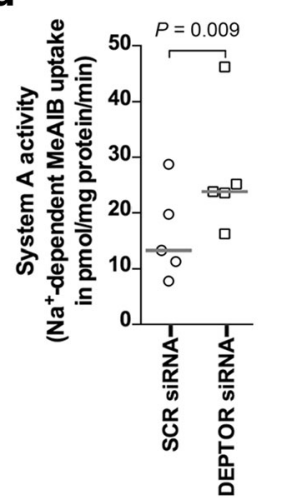

e

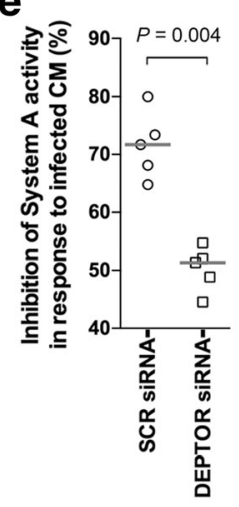

Fig. 3 Constitutive mTOR activation by DEPTOR silencing partially restores System A activity. a Protein expression of DEPTOR (an endogenous mTOR inhibitor) was decreased following DEPTOR silencing in primary human trophoblast cells. mTOR activity was measured as the phosphorylated-to-total protein expression levels of rps6, 4EBP-1, and Akt in primary human trophoblast cells transfected either with DEPTOR siRNA or with a Scramble siRNA control (SCR). b Representative Western blot. DEPTOR silencing resulted in (c) constitutively higher mTORC1 signaling activity only and (d) higher System A activity. e DEPTOR silencing partially restored System A activity in response to infected conditioned medium. $n=5$ placentas. CM conditioned medium, SCR scrambled

associated intervillositis is specifically associated with impaired System A activity and that placental malaria itself (i.e., in the absence of intervillositis) is not associated with impaired System A activity. Also, we showed in our previous publication [12] that infected erythrocytes, either intact or lysed, did not impact on System A activity. These collective observations argue for a limited role (if any) of placental malaria per se on impaired System A activity. As such, we elected not to include a parasite-conditioned medium control. We attempted to model the unique milieu in the intervillous space of women with placental malaria-associated intervillositis using conditioned medium from a co-culture of monocytes and P. falciparum-infected erythrocytes. This conditioned medium displayed high levels of IL-1 $\beta$, IL-6, IL-8, TNF, and IL-10. Cytokines such as IL-1 $\beta$ have been shown to inhibit mTOR activity [28] and similar cytokine profiles have been reported in malariainfected pregnant women [26, 29]. The relevance of our in vitro model is further reinforced by the use of PHT cells, which undergo differentiation to form multinucleated syncytial islands in culture. We recapitulated the findings in women with placental malaria in this cell culture model, providing support for the concept that placental mTOR signaling inhibition mechanistically links placental malariaassociated intervillositis with decreased amino acid uptake.

mTORC1 is the master regulator of the translational machinery and activates protein synthesis by phosphorylating downstream targets including 4EBP-1 (Additional file 1: Table S1). Both in placental tissue obtained from women with placental malaria and in cultured PHT cells, we observed that placental malaria-associated intervillositis consistently inhibited phosphorylation of 4EBP-1 but not that of ribosomal protein S6 (rps6), an indirect target of mTORC1. This may be because 4EBP-1 lies directly downstream of mTORC1, unlike rps6. Reduced 4EBP-1 phosphorylation prevents protein translation initiation, decreasing protein synthesis [30]. Similar to our findings, human fetal growth restriction due to placental insufficiency unrelated to placental malaria is associated with a marked inhibition of 4EBP-1 phosphorylation [16], which could decrease protein synthesis. We speculate that inhibition of placental protein synthesis as a result of inhibition of mTOR signaling further contributes to restricted fetal growth in placental malaria-associated intervillositis.

We also observed that placental malaria-associated intervillositis decreased the phosphorylation of Akt, an 
mTORC2 target, to a greater extent than that of mTORC1 targets. Endoplasmic reticulum (ER) stress inhibits Akt phosphorylation [31, 32] and has been reported in the placenta of non-malaria cases of fetal growth restriction [16]. In placental malaria-associated intervillositis, the inflammatory mediators present in the intervillous space could induce syncytiotrophoblast ER stress [33], which may contribute to placental mTORC2 signaling inhibition [31] in addition to mTOR-dependent mechanisms. Given the strong association between mTORC2 activity and System A activity both ex vivo (Table 2, Additional file 2: Figure S1) and in vitro in PTH cells [20], this greater mTORC2 signaling inhibition could explain the extent of System A inhibition observed in placental malaria-associated intervillositis [12].

We established mTOR signaling as a mechanistic link between placental malaria-associated intervillositis and reduced amino acid uptake using RNAi-mediated silencing of DEPTOR, an endogenous mTOR inhibitor [21]. Knockdown of DEPTOR results in constitutive activation of mTOR signaling [34]. In the current study, DEPTOR silencing in PHT cells resulted in increased mTORC1 but not mTORC2 signaling activity. This is in agreement with Peterson and colleagues who established that knockdown of DEPTOR was sufficient to activate mTORC1 but not mTORC2 [21], causing an asymmetrical effect on mTOR signaling [35]. However, we previously observed that DEPTOR silencing in PHT cells activated both MTORC1 and mTORC2 [13]. Differences in the sequences of DEPTOR siRNA used in these studies might underlie the differing results. Given that placental malaria-associated intervillositis in the placentas of women with placental malaria and in culture PHT cells appears to inhibit mTORC2 more strongly than mTORC1, it is possible that most of the decrease in System A activity in response to placental malaria-associated intervillositis is mediated by mTORC2. The lack of significant effect of DEPTOR silencing on mTORC2 signaling could therefore explain why DEPTOR silencing only partly restored the decrease in System A activity in response to infected conditioned media. Alternatively, the partial restoration could also be due to the incomplete silencing of DEPTOR following DEPTOR siRNA transfection. Regardless, this finding suggests that increasing placental mTOR signaling could upregulate the uptake of amino acids, thereby improving birthweight.

Our results provide evidence for a causal link between placental malaria-associated intervillositis, mTOR signaling inhibition, and decreased amino acid uptake, an important determinant of fetal amino acid availability and fetal growth. The positive correlation between mTOR signaling and birthweight further supports the hypothesis that placental mTOR signaling influences fetal growth. We chose to focus on System A because this amino acid transporter is regulated by mTOR and is the placental transporter system most strongly associated with fetal growth [8]. Further work should investigate whether other placental amino acid transporters are inhibited in placental malariaassociated intervillositis. Further, a detailed profiling of inflammatory mediators in the intervillous blood and the conditioned media will help to identify factors that inhibit placental mTOR signaling.

Our findings identify placental mTOR as a potentially valuable target for interventions aimed at improving birthweight in malaria in pregnancy. There is strong interest in targeting mTOR activity in therapeutic development and various mTOR signaling regulators have recently been tested in clinical trials for other conditions [36]. Some are already used in the clinic [37, 38]. Further, recent studies provide emerging evidence that activators of mTOR signaling can be used to prevent or reverse fetal growth restriction of causes other than placental malaria [39-49]. For example, late gestation arginine treatment of women with unknown causes of fetal growth restriction increased birthweight (177 to 328 g; $\sim 10 \%$ of normal birthweight) and decreased incidence of fetal growth restriction by $40-50 \%$ [41, 43]. Importantly, these interventions were conducted after the diagnosis of fetal growth restriction, suggesting that arginine can rescue fetal growth. Leucine treatment (in combination with other branched-chain amino acids) has also been repeatedly shown to improve fetal growth in various species and models of fetal growth restriction [47-49]. For example, leucine is essential for attenuating fetal growth restriction caused by a protein restriction in rats [47] and restores fetal weight in a mouse model of tumor-induced fetal growth restriction [49]. Significantly, these studies established that leucine supplementation also activated placental mTOR signaling. We propose that restoring placental mTOR signaling in placental malaria would enhance placental amino acid uptake and improve fetal growth and pregnancy outcomes, and should be further investigated. This could be first done using our in vitro model by testing mTOR activators for their capacity to restore amino acid uptake by PHT cells exposed to infected conditioned media. Positive hits could then be further investigated in animal models of malaria in pregnancy [50].

\section{Conclusions}

Various malaria control measures have decreased malaria prevalence but with limited impact on the birthweight of babies born to malaria-infected women. Similarly, modest gains in birthweight have been achieved using untargeted maternal dietary supplementation $[5,6]$. There is an urgent need for a better understanding of the mechanisms leading to low birthweight in malaria in pregnancy. This will allow for the development of new interventions directly aimed at improving birthweight that would be complementary to existing malaria control measures. Here, our data strongly suggest that inflammatory mediators in 


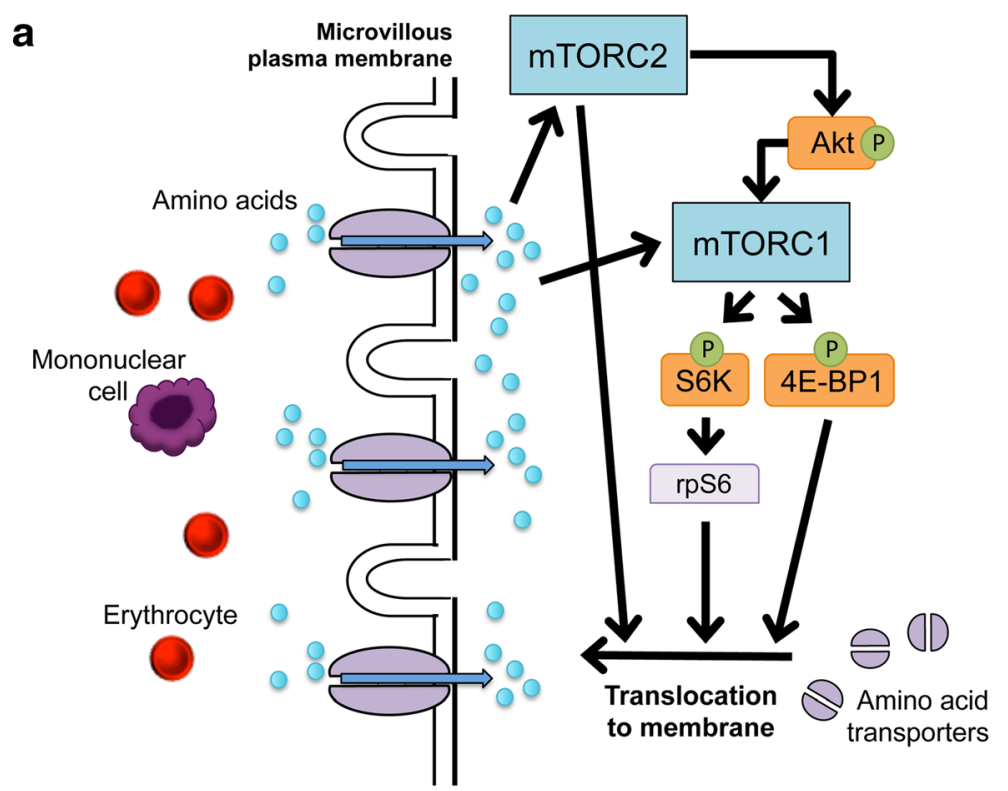

Uninfected placenta: Adequate fetal amino acid supply,
translating to appropriate fetal growth and birthweight

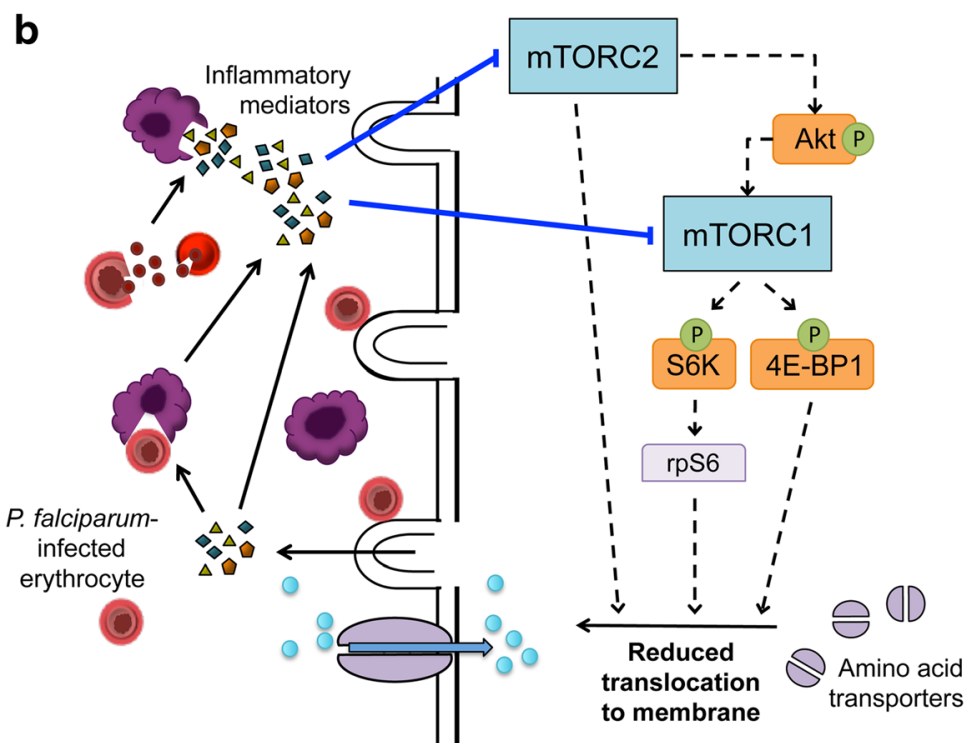

Placental malaria with intervillositis:

mTOR inhibition impairs fetal amino acid supply,

translating to fetal growth restriction and lower birthweight

Fig. 4 Proposed mechanism for reduced birthweight in placental malaria-associated intervillositis. a Under normal physiological conditions, upstream regulators of mTOR such as growth factors and hormones activate mTOR and its downstream effectors rps6, 4E-BP1, and Akt, which promote the translocation of amino acid transporters to the microvillous plasma membrane, promoting transplacental amino acid transfer and adequate fetal growth and birthweight. $\mathbf{b}$ In placental malaria-associated intervillositis, inflammatory mediators are released by monocytes after phagocytosis of $P$. falciparum-infected erythrocytes, by rupture of infected erythrocytes, and by the syncytiotrophoblast in response to the adhesion of infected erythrocytes. This inflammatory response inhibits mTOR signaling, reducing amino acid transport by decreasing the translocation of amino acid transporters to the microvillous plasma membrane. This results in a suboptimal amino acid fetal supply and reduced fetal growth and birthweight 
placental malaria-associated intervillositis inhibit placental mTOR signaling, reducing placental amino acid transport, which is likely to contribute to decreased birthweight (Fig. 4). mTOR activation increases the surface abundance of amino acid transporters in the plasma membranes of the syncytiotrophoblast allowing for increased amino acid transport [13], which may improve fetal growth and birthweight. This identifies placental mTOR as a potentially valuable target for interventions aimed at improving birthweight in malaria in pregnancy.

\section{Additional files}

Additional file 1: Supplementary materials and methods, Table S1, and supplementary figure legends. (DOC $113 \mathrm{~kb}$ )

Additional file 2: Figure S1. Correlation between mTOR signaling activity and the degree of intervillositis, System A activity, and birthweight. (TIFF $491 \mathrm{~kb}$ )

Additional file 3: Figure S2. Cytokine profiles in conditioned media. (TIF $191 \mathrm{~kb}$ )

Additional file 4: Figure S3. Syncytialization and viability of cultured primary human trophoblasts. (TIF $498 \mathrm{~kb}$ )

\section{Abbreviations}

4EBP-1: Eukaryotic translation initiation factor 4E-binding protein 1; Akt: Ak thymoma; CCL: CC chemokine ligand; DEPTOR: DEP domain-containing mTOR-interacting protein; IFN: Interferon; IL: Interleukin; MCP: Monocyte chemotactic protein; MIP: Macrophage inflammatory protein; mTOR: Mechanistic target of rapamycin; mTORC1: mTOR complex 1; mTORC2: mTOR complex 2; PHT: Primary human trophoblast; rpS6: Ribosomal protein 6; Ser: Serine; siRNA: small interfering RNA; Thr: Threonine; TNF: Tumor necrosis factor

\section{Acknowledgements}

Not applicable.

\section{Funding}

This work was supported by The Henry and Rachael Ackman Travelling Scholarship from the Faculty of Medicine, Dentistry and Health Sciences, University of Melbourne, to KGD, by the National Institutes of Health [RHD68370] to TJ, by the Australian NHMRC to JGB, and by the Australian Research Council to FJIF. The Burnet Institute is supported by the National Health and Medical Research Council Independent Research Institutes Infrastructure Support Scheme, and a Victoria State Government Operational Infrastructure Support grant. These funding bodies had no role in the design of the study and collection, analysis, and interpretation of data, and in writing the manuscript.

\section{Availability of data and materia}

The datasets during and/or analyzed during the current study are available from the corresponding author on reasonable request.

\section{Authors' contributions}

KGD performed most of the experimental work and took part in writing the manuscript. EHA generated the conditioned media. FR provided key technical help for western blotting. MN performed the cytokine-bead array assays. JG was a major contributor to System A activity measurements and helped draft the manuscript. SJR coordinated the cohort study in Malawi and helped draft the manuscript. FJIF performed the statistical analyses. JGB was a major contributor to data analysis and interpretation and helped draft the manuscript. TP and TJ supervised the in vitro PHT model work. TJ was key in the design, implementation, and interpretation of the PHT work and had major input in writing the manuscript. PB supervised the study, performed some of the experimental work, and drafted the manuscript. All authors read and approved the final manuscript.

\section{Competing interests}

The authors declare that they have no competing interests.

Consent for publication

Not applicable.

Ethics approval and consent to participate

The College of Medicine Research Ethics Committee, University of Malawi, approved this study. Written informed consent was obtained from primiparous Malawian women. For in vitro work, placental villous tissue samples were collected from healthy women with normal term pregnancies following written informed consent as approved by the Colorado Multiple Institutional Review Board (COMIRB-14-1073).

\section{Author details}

${ }^{1}$ Department of Medicine at Royal Melbourne Hospital, The University of Melbourne, Parkville 3004, VIC, Australia. ${ }^{2}$ Centre for Biomedical Research, Burnet Institute, 85 Commercial Road, Melbourne 3004, VIC, Australia. ${ }^{3}$ Department of Obstetrics \& Gynecology, University of Colorado Anschutz Medical Campus, Aurora, CO, USA. ${ }^{4}$ Maternal and Fetal Health Research Centre, Division of Developmental Biology \& Medicine, School of Medical Sciences, Faculty of Biology, Medicine \& Health, University of Manchester, St. Mary's Hospital, Manchester, UK. ${ }^{5}$ Victorian Infectious Diseases Service, Royal Melbourne Hospital, Parkville, VIC, Australia. ${ }^{6}$ Centre for Epidemiology and Biostatistics, Melbourne School of Population and Global Health, The University of Melbourne, Melbourne, VIC, Australia. ${ }^{7}$ Department of Epidemiology and Preventive Medicine, Department of Infectious Diseases, Monash University, Melbourne, VIC, Australia. ${ }^{8}$ Department of Microbiology and Central Clinical School, Monash University, Clayton 3800, VIC, Australia. ${ }^{9}$ Department of Pediatrics, University of Colorado Anschutz Medical Campus, Aurora, CO, USA.

Received: 17 August 2016 Accepted: 30 November 2016

Published online: 03 January 2017

\section{References}

1. UNICEF. Low birthweight: percentage of infants weighing less than 2,500 grams at birth. New York: UNICEF; 2013.

2. Walker PG, ter Kuile FO, Garske T, Menendez C, Ghani AC. Estimated risk of placental infection and low birthweight attributable to Plasmodium falciparum malaria in Africa in 2010: a modelling study. Lancet Glob Health. 2014;2(8):e460-7.

3. Desai M, ter Kuile FO, Nosten F, McGready R, Asamoa K, Brabin B, Newman $\mathrm{RD}$. Epidemiology and burden of malaria in pregnancy. Lancet Infect Dis. 2007;7(2):93-104.

4. Rogerson SJ, Pollina E, Getachew A, Tadesse E, Lema VM, Molyneux ME. Placental monocyte infiltrates in response to Plasmodium falciparum malaria infection and their association with adverse pregnancy outcomes. Am J Trop Med Hyg. 2003;68(1):115-9.

5. Radeva-Petrova D, Kayentao K, ter Kuile FO, Sinclair D, Garner P. Drugs for preventing malaria in pregnant women in endemic areas: any drug regimen versus placebo or no treatment. Cochrane Database Syst Rev. 2014;10, CD000169.

6. Christian P, Tielsch JM. Evidence for multiple micronutrient effects based on randomized controlled trials and meta-analyses in developing countries. J Nutr. 2012;142(1):173S-7.

7. Roos S, Powell TL, Jansson T. Placental mTOR links maternal nutrient availability to fetal growth. Biochem Soc Trans. 2009;37(Pt 1):295-8.

8. Glazier JD, Cetin I, Perugino G, Ronzoni S, Grey AM, Mahendran D, Marconi AM, Pardi G, Sibley CP. Association between the activity of the system A amino acid transporter in the microvillous plasma membrane of the human placenta and severity of fetal compromise in intrauterine growth restriction. Pediatr Res. 1997;42(4):514-9.

9. Jansson T, Ekstrand Y, Bjorn C, Wennergren M, Powell TL. Alterations in the activity of placental amino acid transporters in pregnancies complicated by diabetes. Diabetes. 2002;51(7):2214-9.

10. Jansson N, Pettersson J, Haafiz A, Ericsson A, Palmberg I, Tranberg M, Ganapathy $V$, Powell TL, Jansson T. Down-regulation of placental transport of amino acids precedes the development of intrauterine growth restriction in rats fed a low protein diet. J Physiol Lond. 2006;576(3):935-46. 
11. Rosario FJ, Jansson N, Kanai Y, Prasad PD, Powell TL, Jansson T. Maternal protein restriction in the rat inhibits placental insulin, mTOR, and STAT3 signaling and down-regulates placental amino acid transporters. Endocrinology. 2011;152(3):1119-29.

12. Boeuf P, Aitken EH, Chandrasiri U, Chua CL, Mclnerney B, McQuade L, Duffy M, Molyneux M, Brown G, Glazier J, et al. Plasmodium falciparum malaria elicits inflammatory responses that dysregulate placental amino acid transport. PLoS Pathog. 2013;9(2), e1003153.

13. Rosario FJ, Dimasuay KG, Kanai Y, Powell TL, Jansson T. Regulation of amino acid transporter trafficking by $\mathrm{mTORC1}$ in primary human trophoblast cells is mediated by the ubiquitin ligase Nedd4-2. Clin Sci (Lond). 2015;130(7):499-512.

14. Jansson T, Aye IL, Goberdhan DC. The emerging role of mTORC1 signaling in placental nutrient-sensing. Placenta. 2012;33 Suppl 2:e23-9.

15. Jansson T, Powell TL. Role of placental nutrient sensing in developmental programming. Clin Obstet Gynecol. 2013;56(3):591-601.

16. Chen $Y Y$, Rosario FJ, Shehab MA, Powell TL, Gupta MB, Jansson T. Increased ubiquitination and reduced plasma membrane trafficking of placental amino acid transporter SNAT-2 in human IUGR. Clin Sci (Lond). 2015;129(12):1131-41.

17. Kliman HJ, Nestler JE, Sermasi E, Sanger JM, Strauss 3rd JF. Purification, characterization, and in vitro differentiation of cytotrophoblasts from human term placentae. Endocrinology. 1986;118(4):1567-82

18. Aye IL, Gao X, Weintraub ST, Jansson T, Powell TL. Adiponectin inhibits insulin function in primary trophoblasts by PPARalpha-mediated ceramide synthesis. Mol Endocrinol. 2014;28(4):512-24.

19. Roos S, Kanai $Y$, Prasad PD, Powell TL, Jansson T. Regulation of placental amino acid transporter activity by mammalian target of rapamycin. Am J Physiol Cell Physiol. 2009;296(1):C142-50.

20. Rosario FJ, Kanai Y, Powell TL, Jansson T. Mammalian target of rapamycin signalling modulates amino acid uptake by regulating transporter cell surface abundance in primary human trophoblast cells. J Physiol Lond. 2013;591(3):609-25.

21. Peterson TR, Laplante M, Thoreen CC, Sancak Y, Kang SA, Kuehl WM, Gray NS, Sabatini DM. DEPTOR is an mTOR inhibitor frequently overexpressed in multiple myeloma cells and required for their survival. Cell. 2009;137(5):873-86.

22. Barker DJP. Maternal nutrition, fetal nutrition, and disease in later life. Nutrition. 1997;13(9):807-13.

23. Jansson T, Powell TL. Human placental transport in altered fetal growth: does the placenta function as a nutrient sensor? A review. Placenta. 2006;27: s91-7.

24. Dimasuay KG, Boeuf $P$, Powell $T$, Jansson T. Placental responses to changes in the maternal environment determine fetal growth. Frontiers in Physiology. 2016;7:12.

25. Suguitan Jr AL, Leke RG, Fouda G, Zhou A, Thuita L, Metenou S, Fogako J, Megnekou R, Taylor DW. Changes in the levels of chemokines and cytokines in the placentas of women with Plasmodium falciparum malaria. J Infect Dis. 2003;188(7):1074-82.

26. Rogerson SJ, Brown HC, Pollina E, Abrams ET, Tadesse E, Lema VM, Molyneux ME. Placental tumor necrosis factor alpha but not gamma interferon is associated with placental malaria and low birth weight in Malawian women. Infect Immun. 2003;71(1):267-70.

27. Ioannidis LJ, Nie CQ, Hansen DS. The role of chemokines in severe malaria: more than meets the eye. Parasitology. 2014;141(5):602-13.

28. Pantham P, Aye IL, Powell TL. Inflammation in maternal obesity and gestational diabetes mellitus. Placenta. 2015;36(7):709-15.

29. Vasquez AM, Segura C, Blair S. Induction of pro-inflammatory response of the placental trophoblast by Plasmodium falciparum infected erythrocytes and TNF. Malar J. 2013;12:421.

30. Thoreen CC, Chantranupong L, Keys HR, Wang T, Gray NS, Sabatini DM. A unifying model for mTORC1-mediated regulation of mRNA translation. Nature. 2012:485(7396):109-13.

31. Yung HW, Charnock-Jones DS, Burton GJ. Regulation of AKT phosphorylation at Ser473 and Thr308 by endoplasmic reticulum stress modulates substrate specificity in a severity dependent manner. Plos One. 2011;6(3), e17894.

32. Chen $\mathrm{CH}$, Shaikenov T, Peterson TR, Aimbetov R, Bissenbaev AK, Lee SW, Wu J, Lin HK, dos Sarbassov D. ER stress inhibits MTORC2 and Akt signaling through GSK-3beta-mediated phosphorylation of rictor. Sci Signal. 2011;4(161):ra10.

33. Brozzi F, Nardelli TR, Lopes M, Millard I, Barthson J, Igoillo-Esteve M, Grieco FA, Villate O, Oliveira JM, Casimir M, et al. Cytokines induce endoplasmic reticulum stress in human, rat and mouse beta cells via different mechanisms. Diabetologia. 2015;58(10):2307-16.
34. Zhao $Y$, Xiong $X$, Sun Y. DEPTOR, an mTOR inhibitor, is a physiological substrate of SCF(betaTrCP) E3 ubiquitin ligase and regulates survival and autophagy. Mol Cell. 2011;44(2):304-16.

35. Proud CG. Dynamic balancing: DEPTOR tips the scales. J Mol Cell Biol. 2009;1(2):61-3.

36. Porta C, Paglino C, Mosca A. Targeting PI3K/Akt/mTOR signaling in cancer Front Oncol. 2014:4:64

37. Yao JC, Shah MH, Ito T, Bohas CL, Wolin EM, Van Cutsem E, Hobday TJ, Okusaka T, Capdevila J, de Vries EG, et al. Everolimus for advanced pancreatic neuroendocrine tumors. N Engl J Med. 2011;364(6):514-23.

38. Hudes G, Carducci M, Tomczak P, Dutcher J, Figlin R, Kapoor A, Staroslawska E, Sosman J, McDermott D, Bodrogi I, et al. Temsirolimus, interferon alfa, or both for advanced renal-cell carcinoma. N Engl J Med. 2007:356(22):2271-81.

39. Kong X, Tan B, Yin Y, Gao H, Li X, Jaeger LA, Bazer FW, Wu G. L-Arginine stimulates the mTOR signaling pathway and protein synthesis in porcine trophectoderm cells. J Nutr Biochem. 2012;23(9):1178-83.

40. Lassala A, Bazer FW, Cudd TA, Datta S, Keisler DH, Satterfield MC, Spencer TE, Wu G. Parenteral administration of L-arginine prevents fetal growth restriction in undernourished ewes. J Nutr. 2010;140(7):1242-8.

41. Sieroszewski P, Suzin J, Karowicz-Bilinska A. Ultrasound evaluation of intrauterine growth restriction therapy by a nitric oxide donor (L-arginine). Matern Fetal Neonatal Med. 2004;15(6):363-6.

42. Wu G, Bazer FW, Satterfield MC, Li X, Wang X, Johnson GA, Burghardt RC, Dai Z, Wang J, Wu Z. Impacts of arginine nutrition on embryonic and fetal development in mammals. Amino Acids. 2013;45(2):241-56.

43. Xiao XM, Li LP. L-Arginine treatment for asymmetric fetal growth restriction. Int J Gynaecol Obstet. 2005;88(1):15-8.

44. Bourdon A, Parnet P, Nowak C, Tran NT, Winer N, Darmaun D. L-citrulline supplementation enhances fetal growth and protein synthesis in rats with intrauterine growth restriction. J Nutr. 2016;146(3):532-41.

45. Sawant OB, Wu G, Washburn SE. Maternal L-glutamine supplementation prevents prenatal alcohol exposure-induced fetal growth restriction in an ovine model. Amino Acids. 2015;47(6):1183-92.

46. Wu G, Bazer FW, Johnson GA, Knabe DA, Burghardt RC, Spencer TE, Li XL, Wang JJ. Triennial Growth Symposium: important roles for L-glutamine in swine nutrition and production. J Anim Sci. 2011;89(7):2017-30.

47. Teodoro GF, Vianna D, Torres-Leal FL, Pantaleao LC, Matos-Neto EM, Donato $\mathrm{Jr} \mathrm{J}$, Tirapegui J. Leucine is essential for attenuating fetal growth restriction caused by a protein-restricted diet in rats. J Nutr. 2012;142(5):924-30.

48. Zheng C, Huang C, Cao Y, Wang J, Dong B. Branched-chain amino acids reverse the growth of intrauterine growth retardation rats in a malnutrition model. Asian-Aust J Anim Sci. 2009:22(11):1495-503.

49. Viana LR, Gomes-Marcondes MC. A leucine-rich diet modulates the tumorinduced down-regulation of the MAPK/ERK and PI3K/Akt/mTOR signaling pathways and maintains the expression of the ubiquitin-proteasome pathway in the placental tissue of NMRI mice. Biol Reprod. 2015:92(2):49.

50. Hviid L, Marinho CR, Staalsoe T, Penha-Goncalves C. Of mice and women: rodent models of placental malaria. Trends Parasitol. 2010;26(8):412-9.

\section{Submit your next manuscript to BioMed Central and we will help you at every step:}

- We accept pre-submission inquiries

- Our selector tool helps you to find the most relevant journal

- We provide round the clock customer support

- Convenient online submission

- Thorough peer review

- Inclusion in PubMed and all major indexing services

- Maximum visibility for your research

Submit your manuscript at www.biomedcentral.com/submit
) Biomed Central 